\title{
Global transcriptional analysis of Mycoplasma hyopneumoniae following exposure to hydrogen peroxide
}

\author{
Correspondence \\ F. Chris Minion \\ fcminion@iastate.edu
}

Received 6 July 2007

Revised 7 August 2007

Accepted 9 August 2007
Erin R. Schafer, Michael J. Oneal, Melissa L. Madsen and F. Chris Minion

Department of Veterinary Microbiology and Preventive Medicine, lowa State University, Ames, IA 50011, USA

\begin{abstract}
Mycoplasma hyopneumoniae, the causative agent of swine enzootic pneumonia, colonizes the cilia of swine lungs, causing ciliostasis and cell death. $M$. hyopneumoniae is a component of the porcine respiratory disease complex (PRDC) and is especially problematic for the finishing swine industry, causing the loss of hundreds of millions of dollars in farm revenues worldwide. For successful infection, $M$. hyopneumoniae must effectively resist oxidative stresses due to the release of oxidative compounds from neutrophils and macrophages during the host's immune response. However, the mechanism that $M$. hyopneumoniae uses to avert the host response is still unclear. To gain a better understanding of the transcriptional responses of $M$. hyopneumoniae under oxidative stress, cultures were grown to early exponential phase and exposed to $0.5 \%$ hydrogen peroxide for $15 \mathrm{~min}$. RNA samples from these cultures were collected and compared to RNA samples from control cultures using two-colour PCR-based M. hyopneumoniae microarrays. This study revealed significant downregulation of important glycolytic pathway genes and gene transcription proteins, as well as a protein known to activate oxidative stressor cascades in neutrophils. Sixty-nine per cent of the upregulated genes were hypothetical with no known function. This study has also revealed significantly differentially expressed genes common to other environmental stress responses, indicating that further investigation of universal stress response genes of $M$. hyopneumoniae is merited.
\end{abstract}

\section{INTRODUCTION}

Porcine enzootic pneumonia, an endemic disease in swine populations, infects 200 million pigs every year, causing hundreds of millions of dollars of loss for swine farmers worldwide. The disease is caused by Mycoplasma hyopneumoniae and is a mild but chronic pneumonia characterized by low mortality but high morbidity (Messier et al., 1990). The most common symptom of the disease is a dry, nonproductive cough but other symptoms include fever, impaired growth and the presence of lung tissue lesions. $M$. hyopneumoniae infects swine via aerosol transmission and direct contact; it attaches to the cilia of the tracheal epithelial cells, causing a reduction in ciliary action (DeBey \& Ross, 1994; Zielinski \& Ross, 1993). It affects the immune response and reduces the ability of the host's immune system to fight secondary pathogens such as Pasteurella multocida (Amass et al., 1994) and porcine reproductive and respiratory syndrome virus (PRRSV) (Thacker et al., 1999). M. hyopneumoniae and PRRSV, along with other agents, contribute to the porcine

The microarray data for this paper are available via the Gene Expression Omnibus (http://www.ncbi.nlm.nih.gov/geo) under accession number GSE8304. respiratory disease complex (PRDC), a serious health concern in swine populations worldwide. The damage to lung tissue caused by both enzootic pneumonia and PRDC is the result of the host's immune response to infection.

M. hyopneumoniae actively suppresses the humoral and cellular immune systems of the host during early stages of pneumonia by inhibiting macrophage-mediated phagocytosis and B-cell antibody production. After colonization of the cilia of the host, lymphocytes infiltrate the site of infection, recruiting macrophages, neutrophils and other cellular immune responses. The response of the host immune system causes the lesions seen in the lung tissue of infected swine by increasing phagocytic and cytotoxic activities of macrophages and initiating the chronic inflammatory response (Sarradell et al., 2003). There is also an increased production of IgG in swine infected with $M$. hyopneumoniae, leading to a greater recruitment of macrophages. Increased production of tumour necrosis factor and interleukin- 1 in an infected host also leads to a greater recruitment of neutrophils (Baskerville, 1972; Sarradell et al., 2003). Once recruited to the site of infection, macrophages and neutrophils use superoxides, such as hydrogen peroxide, nitric oxide and superoxide anions, to kill M. hyopneumoniae during respiratory bursts. 
Therefore, it is important for the pathogen to employ mechanisms to resist oxidative stress from the immune response.

Oxidative stress toxicity in bacteria is due in large part to irreversible damage to the organism's DNA (Gusarov \& Nudler, 2005). This is particularly important for organisms with rudimentary DNA repair systems like mycoplasmas, which may be ineffective in repairing this type of DNA damage (Zou \& Dybvig, 2002). Hydrogen peroxide as a byproduct of host respiration or the host's innate immune defence can interact with free cellular iron and form hydroxyl radicals that have the potential to react with DNA bases and sugar moieties. These interactions can cause modifications in the DNA including strand breaks (Gusarov \& Nudler, 2005). The mechanisms that $M$. hyopneumoniae uses in defence against superoxides and other host immune responses are not entirely known. By using hydrogen peroxide to mimic a respiratory burst attack by macrophages and neutrophils, one can examine the effects of respiratory burst on the transcriptome of the organism. The current study examined the transcriptome of $M$. hyopneumoniae under oxidative stress using microarray technology. Differentially expressed genes identified in this study contribute to understanding the mechanism by which $M$. hyopneumoniae evades the host immune system.

\section{METHODS}

Mycoplasma strain and culture conditions. Pathogenic $M$. hyopneumoniae strain 232, a derivative of strain 11 , was used in this study. It can be obtained from Dr Eileen L. Thacker (Iowa State University) or a clone of the parent strain can be obtained from the American Type Culture Collection (ATCC 27714). Cultures were initially inoculated with lung inoculum, and passaged as previously described (Madsen et al., 2006a). Twelve $250 \mathrm{ml}$ flasks containing $150 \mathrm{ml}$ culture were grown to early exponential phase as determined by colour-changing units. Hydrogen peroxide was added to six flasks to a concentration of $0.5 \%$, and all twelve flasks were incubated at $37{ }^{\circ} \mathrm{C}$ for $15 \mathrm{~min}$. Cells were pelleted and stored at $-70{ }^{\circ} \mathrm{C}$ in RNALater (Ambion) as previously described (Madsen et al., 2006a).

Microarray. The M. hyopneumoniae microarray consists of 632 PCRamplified M. hyopneumoniae ORF gene products $125-350 \mathrm{bp}$ in length. These products encompass $91 \%(632 / 698)$ of the ORFs in the genome. The construction and validation of these arrays have been previously described (Madsen et al., 2006a). Each slide was divided into two regions (upper and lower), both of which contained a full array of probes and could be hybridized independently. Each feature (PCR product) was spotted in triplicate in each of the arrays in widely spaced spots onto Corning UltraGAPS glass substrates. Slides were UV cross-linked at $450 \mathrm{~mJ}$ and pre-hybridized with sodium borohydride to reduce background (Raghavachari et al., 2003).

Experimental design. Six independent RNA samples from hydrogen peroxide-exposed cultures were paired with six independent RNA samples from control cultures for hybridization to six two-colour microarrays. For three arrays, the control RNA sample was labelled with Cy3 dye and the experimental RNA sample was labelled with Cy5 dye; the dyes were reversed for the other three arrays to account for any possible dye bias.
RNA isolation. RNA was isolated from frozen cell pellets using the Versagene RNA Purification System (Qiagen). The manufacturer's protocol was followed with the exception of the DNase treatment, which was extended to $30 \mathrm{~min}$. The filter cutoff of $150 \mathrm{bp}$ prevented small RNA fragments from interfering in subsequent manipulations.

Target generation and hybridization. Fluorescently labelled cDNA targets were generated and purified using a modified protocol as described by Boyce et al.(2004) with a 129 hexamer primer set specific for M. hyopneumoniae ORFs used in previous studies (Madsen et al., 2006a). After cDNA target purification, they were hybridized to the array using the previously described protocol (Madsen et al., 2006a). Slides were washed using the Corning UltraGAPS slide wash protocol and dried by centrifugation at $1500 \mathrm{~g}$ for $2 \mathrm{~min}$.

Data acquisition and normalization. After hybridization and washing, the arrays were scanned using a ScanArray Express laser scanner (Applied Biosystems) three times with varying photomultiplier tube gain settings to encompass the dynamic range of the signal intensities (Dudley et al., 2002). Slide images were analysed using the SoftWor ${ }_{\mathrm{x}}$ Tracker package (Applied Precision), acquiring mean spot intensity and median background intensity for each spot. Spot signals were corrected for background by subtracting the median background intensity from the mean spot intensity. The natural logarithms of these background-corrected signals were adjusted by an additive constant to give a common median to each scan of the same arraydye combination. The data from both dye channels on a given array were normalized by locally weighted scatterplot smoother (LOWESS) normalization. These normalized datasets were then adjusted by an additive constant to make each median the same across all arrays. The triplicate spots were averaged together to produce one normalized measurement for each probe on the array.

Data analysis. A separate mixed linear model analysis was conducted for each probe sequence using the normalized data (Wolfinger et al., 2001). Each model included fixed effects for treatment (hydrogen peroxide vs control), array location on slide (upper vs lower) and dye (Cy3 vs Cy5) as well as random effects for slide-slide and region effects. Student $t$-tests for differential expression between treatments were conducted for each probe in the mixed linear model analyses. $q$ values were calculated based on the $P$ values by the method of Storey \& Tibshirani (2003). These $q$ values were used to approximate the false discovery rate (FDR) for any given $P$ value as described by Mosig et al. (2001). Fold changes of expression between treatments were also estimated for each probe by taking the inverse natural logarithm of the estimated mean treatment difference from our mixed linear model analyses.

qRT-PCR. Quantitative reverse transcriptase-polymerase chain reactions (qRT-PCR) were performed as described by the manufacturer. Five genes were analysed using the Brilliant SYBR Green QPCR Master Mix (Stratagene). In addition, gene $m h p 345$ was chosen as the housekeeping gene for these studies based on its consistent expression levels in several microarray studies (Madsen et al., 2006a, b). Cycling conditions were based upon the manufacturer's recommendations (Stratagene). A Bio-Rad MyiQ real-time PCR cycler was used. In all cases the same RNA samples were used for both the microarray and the qRT-PCR studies. The primers used for amplification were mhp008 (forward, 5' -TGGATGGTACAAGCAAAGGTGGGA; reverse,

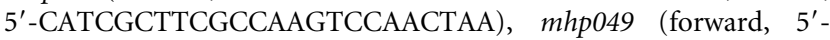
GTTTGGCCTTTGTTAGTTGGTTTGG; reverse, 5'-GAAATTAACGGCGCTGGAATCCCT), mhp061 (forward, 5'-ACTACGGGAGCTAGGGTTTGTT; reverse, 5' -CAGATGCCTGTCAAACTTTCGGGT), $m h p 239$ (forward, 5'-TTTGGCCGTGAATCTACCGGCATT; reverse, 5'-GAACGAGCTTTCGCGATCATTGGT), $m h p 456$ (forward, 5'-CTGATGAAGTATTAGGTAAATTGGCAGAGC; reverse, $5^{\prime}$-CATCGCCATCTTCTTGAATTTCCC) and $m h p 345$ (forward, 
5'-TGAAGCGCTTATGCTACTGAGG; reverse, 5' -ATTGCGGTTGTACGAGCGACCTTA). The qRT-PCR data were analysed according to Gallup \& Ackermann (2006).

\section{RESULTS AND DISCUSSION}

Our studies used hydrogen peroxide exposure as a substitute for oxidative stress encountered in vivo when M. hyopneumoniae infects a host (Farr \& Kogoma, 1991; Storz \& Toledano, 1994; Zheng et al., 2001). Preliminary studies measuring quantitative effects following exposure to hydrogen peroxide on traits such as viability are problematic with $M$. hyopneumoniae since it does not grow well on agar surfaces. Viability of M. hyopneumoniae is measured by colour-changing units in broth using twofold dilutions and is subject to dilution errors. Consequently, a hydrogen peroxide concentration based on previous studies with bacterial pathogens was used (Zheng et al., 2001). Also, unpublished studies by C.-U. R. Zimmerman and R. Herrmann reported at the 16th International Congress of the International Organization for Mycoplasmology (abstract 162) showed that a $0.5 \%$ hydrogen peroxide concentration gave maximal transcriptional changes with minimal cell death in Mycoplasma pneumoniae. In our study, transcriptional changes were measured at $15 \mathrm{~min}$ post exposure based on previous studies with heat shock and iron depletion (Madsen et al., $2006 \mathrm{a}, \mathrm{b})$, both of which showed significant transcript differences at this time point. This time frame was also chosen by Zimmerman and Herrmann in their studies with M. pneumoniae.

Microarrays were used to compare RNA steady-state levels in M. hyopneumoniae strain 232 following exposure to hydrogen peroxide. Post-purification RNA amounts were approximately $2-8 \mu \mathrm{g}$, which was used to generate $1.5-$ $6 \mu \mathrm{g}$ cDNA for labelling reactions. Following labelling reactions, fluorescent labelling efficiencies were 100-150 bp

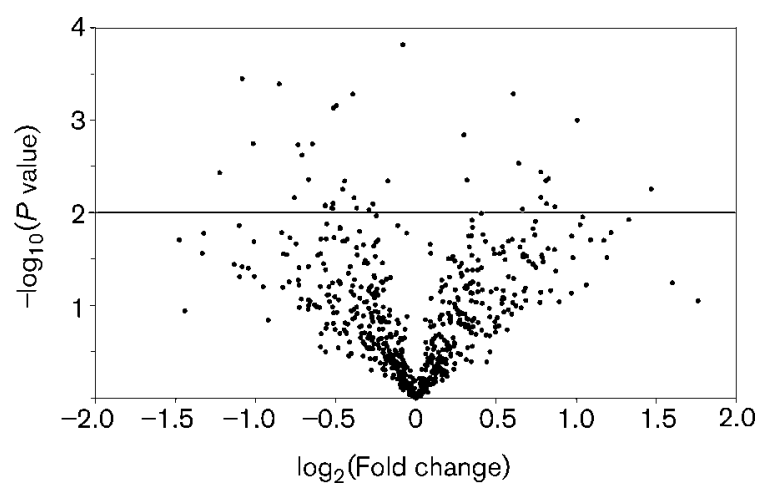

Fig. 1. Volcano plot of transcriptional differences in M. hyopneumoniae during treatment with hydrogen peroxide. Data represent individual gene responses plotted as $\log _{2}$ (fold change) vs $-\log _{10}(P$ value). Points above $-\log _{10} P$ value $=2$ (horizontal line) are significant at $P<0.01$, with a negative fold change representing the downregulated genes and a positive fold change representing the upregulated genes.

per dye for both treatments, which is within the recommended range of label incorporation (http://probes. invitrogen.com/resources/calc/basedyeratio.html). Six arrays were analysed for transcriptional changes.

A mixed linear model design was used to analyse the quantified data from the scanned images. Statistical analysis indicated that 38 genes had significant transcriptional differences with $P<0.01$, with an estimated false discovery rate of $q \leqslant 0.0529$. A volcano plot visualizes the results (Fig. 1). Thirteen genes (Table 1) were found to have higher transcript levels and twenty-five genes (Table 2) were found to have lower transcript levels after exposure to hydrogen peroxide. Five genes were chosen for real-time PCR analysis and all confirmed our microarray data in the direction of regulation (Tables 1 and 2). In most cases, the

Table 1. Upregulated genes following exposure of $M$. hyopneumoniae to hydrogen peroxide

\begin{tabular}{|lllcccc|}
\hline ID & \multirow{2}{*}{ Gene } & \multicolumn{1}{c}{ Description } & P value & q value & \multicolumn{2}{c|}{ Fold change } \\
\cline { 5 - 7 } & & & & & Array & qRT-PCR \\
\hline mhp004 & $\mathrm{CH}$ & Conserved hypothetical & 0.0010 & 0.0270 & 2.01 & \\
mhp024 & $\mathrm{UH}$ & Unique hypothetical & 0.0068 & 0.0529 & 1.72 & \\
mhp049 & atpB & ATP synthase A chain & 0.0014 & 0.0332 & 1.23 & 5.40 \\
mhp061 & glyS & Glycyl-tRNA synthetase & 0.0029 & 0.0447 & 1.56 & 5.08 \\
mhp232 & $\mathrm{UH}$ & Unique hypothetical & 0.0044 & 0.0447 & 1.25 & \\
mhp239 & spoU & tRNA/rRNA methyl transferase & 0.0005 & 0.0226 & 1.52 & 4.82 \\
mhp318 & mglA & ATP-binding protein & 0.0042 & 0.0447 & 1.78 & \\
mhp325 & $\mathrm{CH}$ & Conserved hypothetical & 0.0045 & 0.0447 & 1.75 & \\
mhp326 & $\mathrm{UH}$ & Unique hypothetical & 0.0036 & 0.0447 & 1.72 & \\
mhp355 & $\mathrm{CH}$ & Conserved hypothetical & 0.0079 & 0.0529 & 1.76 & \\
mhp366 & $\mathrm{CH}$ & Conserved hypothetical & 0.0055 & 0.0499 & 2.77 & \\
mhp516 & $\mathrm{CH}$ & Conserved hypothetical & 0.0086 & 0.0529 & 1.82 & \\
mhp623 & $\mathrm{CH}$ & Conserved hypothetical & 0.0090 & 0.0529 & 1.58 & \\
& & & & & & \\
\hline
\end{tabular}


Table 2. Downregulated genes following exposure of $M$. hyopneumoniae to hydrogen peroxide

\begin{tabular}{|c|c|c|c|c|c|c|}
\hline \multirow[t]{2}{*}{ ID } & \multirow[t]{2}{*}{ Gene } & \multirow[t]{2}{*}{ Description } & \multirow[t]{2}{*}{$P$ value } & \multirow[t]{2}{*}{$q$ value } & \multicolumn{2}{|c|}{ Fold change } \\
\hline & & & & & Array & qRT-PCR \\
\hline mhp008 & $f t s Y$ & Cell division protein & 0.0080 & 0.0529 & 1.20 & 1.25 \\
\hline mhp015 & $r l u C$ & $\begin{array}{l}\text { Ribosomal large subunit pseudouridine } \\
\text { synthase }\end{array}$ & 0.0083 & 0.0529 & 1.48 & \\
\hline mhp029 & gatA & Glutamyl-tRNA amidotransferase subunit A & 0.0045 & 0.0447 & 1.13 & \\
\hline mhp038 & $\mathrm{CH}$ & Conserved hypothetical & 0.0093 & 0.0529 & 1.23 & \\
\hline mhp115 & $\mathrm{CH}$ & $\mathrm{ABC}$ transporter ATP-binding protein & 0.0007 & 0.0228 & 1.43 & \\
\hline mhp188 & rpl4 & 50 S ribosomal protein $\mathrm{L} 4$ & 0.0017 & 0.0332 & 2.02 & \\
\hline mhp189 & rpl23 & 50 S ribosomal protein L23 & 0.0004 & 0.0226 & 1.81 & \\
\hline mhp262 & $\mathrm{UH}$ & Unique hypothetical & 0.0069 & 0.0529 & 1.69 & \\
\hline mhp269 & $p f k A$ & 6-Phosphofructokinase & 0.0036 & 0.0447 & 2.34 & \\
\hline mhp293 & $\mathrm{UH}$ & Unique hypothetical & 0.0045 & 0.0447 & 1.36 & \\
\hline $\operatorname{mhp} 383$ & $\mathrm{UH}$ & Hypothetical protein & 0.0001 & 0.0226 & 1.06 & \\
\hline mhp403 & $\mathrm{UH}$ & Unique hypothetical & 0.0079 & 0.0529 & 1.43 & \\
\hline mhp424 & $\mathrm{CH}$ & Conserved hypothetical & 0.0023 & 0.0396 & 1.64 & \\
\hline mhp433 & trmU & $\begin{array}{l}\text { tRNA-(5-methylaminomethyl-2-thiouridylate) } \\
\text { methyltransferase }\end{array}$ & 0.0055 & 0.0499 & 1.37 & \\
\hline mhp445 & $\mathrm{CH}$ & Conserved hypothetical & 0.0017 & 0.0332 & 1.56 & \\
\hline mhp451 & $\mathrm{CH}$ & Conserved hypothetical & 0.0003 & 0.0226 & 2.12 & \\
\hline mhp452 & $\mathrm{CH}$ & Conserved hypothetical & 0.0084 & 0.0529 & 1.48 & \\
\hline mhp456 & napA & Neutrophil-activating protein & 0.0043 & 0.0447 & 1.59 & 5.79 \\
\hline mhp495 & $y x 1$ & Conserved hypothetical & 0.0007 & 0.0228 & 1.41 & \\
\hline mhp508 & $\mathrm{UH}$ & Unique hypothetical & 0.0089 & 0.0529 & 1.43 & \\
\hline mhp545 & gyrA & DNA gyrase subunit A & 0.0005 & 0.0226 & 1.31 & \\
\hline mhp561 & $\mathrm{CH}$ & Conserved hypothetical & 0.0089 & 0.0529 & 1.29 & \\
\hline mhp630 & $\mathrm{CH}$ & Conserved hypothetical & 0.0069 & 0.0529 & 1.30 & \\
\hline mhp662 & $\mathrm{CH}$ & Conserved hypothetical & 0.0088 & 0.0529 & 1.44 & \\
\hline mhp672 & rplM & 50S ribosomal protein L13 & 0.0018 & 0.0332 & 1.66 & \\
\hline
\end{tabular}

qRT-PCR results showed greater increases or decreases than the microarray data.

Previous studies measured transcriptional changes during other environmental stresses (heat shock and iron depletion) in M. hyopneumoniae (Madsen et al., 2006a, b). Since oxygen radicals also cause stress, particularly in relation to DNA, it was of interest to determine if there were genes responsive to all three treatments. These genes could then be categorized as general stress genes of $M$. hyopneumoniae. Only two genes were regulated in all three studies (mhp325 and mhp672). Ten genes were regulated both by heat shock and in this study (mhp008, mhp015, mhp029, mhp038, mhp061, mhp232, mhp269, mhp325, mhp445 and mhp672). Four genes were regulated both by iron deprivation and in this study (mhp188, mhp239, mhp325 and mhp672).

Genes common to the heat-shock study and this study include translation and DNA replication genes such as $r l u C$ (mhp015) and rplM (mhp672). These were both downregulated, indicating that oxidative stress, like heat shock, results in lower levels of translation and DNA replication. It is thought that this allows the cell to focus its energy on remaining viable and not on cell division. dnaK was upregulated in the hydrogen peroxide-treated group
( $P=0.0118)$. Madsen et al. (2006a) identified $d n a K$ as the most significant upregulated gene in the heat-shock study, and it is reasonable to infer that the importance of this chaperone in protecting against protein misfolding applies not only during heat shock but also during oxidative stress. It is evident that $M$. hyopneumoniae responds to different types of stress in a manner that has some commonality.

Of the thirteen genes found to be upregulated during oxidative stress, nine (69\%) have no assigned function (mhp004, mhp024, mhp232, mhp325, mhp326, mhp366, mhp355, mhp516 and mhp623). Further study will be necessary to eventually assign function. The upregulated genes with assigned function include atpB (mhp049), glyS (mhp061), spoU (mhp239) and mglA (mhp318). spoU and glyS have assigned functions within tRNA synthesis and regulation, indicating that oxidative stress leads to an increase in protein synthesis. The increased levels of atpB and $m g l A$ transcripts indicate the elevated use of and need for ATP during oxidative damage, possibly in repair of damaged cellular components.

Oxidative stress caused downregulation in 25 genes in $M$. hyopneumoniae. Despite the upregulation of spoU and $g l y S$, genes associated with ribosomal assembly and processing including rpl23 (mhp189), rplM (mhp672), rpl4 (mhp188) 
and $\mathrm{rluC}(\mathrm{mph} 015)$ were downregulated. Although this seems paradoxical, oxidative radical damage seems to interfere with ribosome synthesis to conserve energy, an activity that consumes a disproportionate amount of ATP. DNA synthesis and cell growth were also slowed when $M$. hyopneumoniae was treated with hydrogen peroxide, as seen in the downregulation of gyrA (mhp545), a gene encoding DNA gyrase subunit A, and ftsY (mhp008), a gene involved in cell division. Thus, M. hyopneumoniae seems to be concentrating its energy demands away from DNA synthesis and cell division. pkfA (mhp269), the gene encoding 6-phosphofructokinase, was also downregulated. This is interesting as 6-phosphofructokinase catalyses the committed step of glycolysis. The downregulation of this gene is an example of suppressing cellular metabolism to direct energy where it is most needed.

Another interesting downregulated gene was napA (mhp456), thought to be responsible for activation of neutrophils. In Helicobacter pylori NapA plays a dual role in pathogenesis: in colonization and in protection against oxidative damage (Wang et al., 2006). As a protein that binds iron, it may thus serve as a primitive siderophore for mycoplasmas, although in previous work, napA was not transcriptionally responsive to changes in iron concentration in M. hyopneumoniae (Madsen et al., 2006b) as has been shown in other bacterial pathogens (Mey et al., 2005). The regulation of this gene may be a clear example of a mechanism M. hyopneumoniae uses to resist the host immune response by not activating neutrophils and thus decreasing the amount of oxidative radicals.

Other downregulated genes included gatA (mhp029) and trmU (mhp433), both of which function as transferases involved in protein processing and synthesis. In addition, fourteen genes with no known function were downregulated. Further research is needed to determine the function of these genes.

Interestingly, no genes for DNA-repair enzymes were regulated during oxidative damage. M. hyopneumoniae, like all mycoplasmas, has limited DNA repair capabilities (Zou \& Dybvig, 2002). The lack of regulation of these activities in M. hyopneumoniae during oxidative damage may indicate that its normal environment, the swine lung, requires constitutive expression of these activities for survival. Mycoplasmas generally do not survive for long periods outside the host. In fact, they spend their lives in highly competitive environments within the host. Therefore, gene regulation may be more subtle in mycoplasmas as opposed to other pathogens, where gene transcription control is often dramatic within markedly different environments.

Many of the mechanisms employed by M. hyopneumoniae to cause disease are still unclear and many relevant genes still have no assigned functions. This study identified the transcriptional response of M. hyopneumoniae to oxidative stress. Since this is only one component of the host immune response to infection, additional studies of other innate immune system onslaughts may eventually increase our understanding of avoidance mechanisms and lead to better drug development by targeting specific gene products, alleviating economic losses in the swine industry.

\section{ACKNOWLEDGEMENTS}

This work was supported in part by a University Honors Program Stewart Research Award. We thank Dr Eileen L. Thacker and members of her laboratory (Nancy Upchurch and Barb Erickson) for the mycoplasma growth media. We thank Stuart Gardner for assistance with data submission.

\section{REFERENCES}

Amass, S. F., Clark, L. K., van Alstine, W. G., Bowersock, T. L., Murphy, D. A., Knox, K. E. \& Albregts, S. R. (1994). Interaction of Mycoplasma hyopneumoniae and Pasteurella multocida infections in swine. J Am Vet Med Assoc 204, 102-107.

Baskerville, A. (1972). Development of the early lesions in experimental enzootic pneumonia of pig: an ultrastructural and histological study. Res Vet Sci 13, 570-578.

Boyce, J. D., Wilkie, I., Harper, M., Paustian, M. L., Kapur, V. \& Adler, B. (2004). Genomic-scale analysis of Pasteurella multocida gene expression during growth within liver tissue of chickens with fowl cholera. Microbes Infect 6, 290-298.

DeBey, M. C. \& Ross, R. F. (1994). Ciliostasis and loss of cilia induced by Mycoplasma hyopneumoniae in porcine tracheal organ cultures. Infect Immun 62, 5312-5318.

Dudley, A. M., Aach, J., Steffen, M. A. \& Church, G. M. (2002). Measuring absolute expression with microarrays with a calibrated reference sample and an extended signal intensity range. Proc Natl Acad Sci U S A 99, 7554-7559.

Farr, S. B. \& Kogoma, T. (1991). Oxidative stress responses in Escherichia coli and Salmonella typhimurium. Microbiol Rev 55, 561-585.

Gallup, J. M. \& Ackermann, M. R. (2006). Addressing fluorogenic realtime qPCR inhibition using the novel custom Excel file system 'Focusfield2-6GallupqPCRSet-upTool-001' to attain consistently high fidelity qPCR reactions. Biol Proced Online 8, 87-152.

Gusarov, I. \& Nudler, E. (2005). NO-mediated cytoprotection: instant adaptation to oxidative stress in bacteria. Proc Natl Acad Sci U S A 102, 13855-13860.

Madsen, M. L., Nettleton, D., Thacker, E. L., Edwards, R. \& Minion, F. C. (2006a). Transcriptional profiling of Mycoplasma hyopneumoniae during heat shock using microarrays. Infect Immun 74, 160-166.

Madsen, M. L., Nettleton, D., Thacker, E. L. \& Minion, F. C. (2006b). Transcriptional profiling of Mycoplasma hyopneumoniae during iron depletion using microarrays. Microbiology 152, 937-944.

Messier, S., Ross, R. F. \& Paul, P. S. (1990). Humoral and cellular immune responses of pigs inoculated with Mycoplasma hyopneumoniae. Am J Vet Res 51, 52-58.

Mey, A. R., Wyckoff, E. E., Kanukurthy, V., Fisher, C. R. \& Payne, S. M. (2005). Iron and Fur regulation in Vibrio cholerae and the role of Fur in virulence. Infect Immun 73, 8167-8178.

Mosig, M. O., Lipkin, E., Galina, K., Tchourzyna, E., Soller, M. \& Friedmann, A. (2001). A whole genome scan for quantitative trait loci affecting milk protein percentage in Israeli-Holstein cattle, by means of selective milk DNA pooling in a daughter design, using an adjusted false discovery rate criterion. Genetics 157, 1683-1698. 
Raghavachari, N., Bao, Y. P., Li, G., Xie, X. \& Mèuller, U. R. (2003). Reduction of autofluorescence on DNA microarrays and slide surfaces by treatment with sodium borohydride. Anal Biochem 312, 101-105.

Sarradell, J., Andrada, M., Ramâirez, A. S., Fernâandez, A., GâomezVillamandos, J. C., Jover, A., Lorenzo, H., Herrâaez, P. \& Rodrâiguez, F. (2003). A morphologic and immunohistochemical study of the bronchus-associated lymphoid tissue of pigs naturally infected with Mycoplasma hyopneumoniae. Vet Pathol 40, 395-404.

Storey, J. D. \& Tibshirani, R. (2003). Statistical significance for genomewide studies. Proc Natl Acad Sci U S A 100, 9440-9445.

Storz, G. \& Toledano, M. B. (1994). Regulation of bacterial gene expression in response to oxidative stress. In Bacterial Pathogenesis, Part B, Interaction of Pathogenic Bacteria with Host Cells, pp. 196207. Edited by V. L. Clark \& P. M. Bavoil. New York: Academic Press.

Thacker, E. L., Halbur, P. G., Ross, R. F., Thanawongnuwech, R. \& Thacker, B. J. (1999). Mycoplasma hyopneumoniae potentiation of porcine reproductive and respiratory syndrome virus-induced pneumonia. J Clin Microbiol 37, 620-627.
Wang, G., Hong, Y., Olczak, A., Maier, S. E. \& Maier, R. J. (2006). Dual roles of Helicobacter pylori NapA in inducing and combating oxidative stress. Infect Immun 74, 6839-6846.

Wolfinger, R. D., Gibson, G., Wolfinger, E. D., Bennett, L., Hamadeh, H., Bushel, P., Afshari, C. \& Paules, R. S. (2001). Assessing gene significance from cDNA microarray expression data via mixed models. J Comput Biol 8, 625-637.

Zheng, M., Wang, X., Templeton, L. J., Smulski, D. R., LaRossa, R. A. \& Storz, G. (2001). DNA microarray-mediated transcriptional profiling of the Escherichia coli response to hydrogen peroxide. J Bacteriol 183, 4562-4570.

Zielinski, G. C. \& Ross, R. F. (1993). Adherence of Mycoplasma hyopneumoniae to porcine ciliated respiratory tract cells. Am J Vet Res 54, 1262-1269.

Zou, N. \& Dybvig, K. (2002). DNA replication, repair and stress response. In Molecular Biology and Pathogenicity of Mycoplasmas, pp. 303-321. Edited by S. Razin \& R. Herrmann. New York: Kluwer Academic/Plenum.

Edited by: G. Firrao 